\title{
Gradient Feature Based Improved Optimum Non-Negative Integer Bit Allocation for the DCT Based Image Coding
}

\author{
Vikrant Singh Thakur ${ }^{1}$, Shubhrata Gupta ${ }^{2}$, Kavita Thakur ${ }^{3}$, K. R. Rao ${ }^{4}$ \\ ${ }^{1}$ Department of Electrical Engg., National Institute of Technology, Raipur, India, Email: vikrant.st@ gmail.com \\ ${ }^{2}$ Department of Electrical Engg., National Institute of Technology, Raipur, India, Email: sgupta.ele@ nitrr.ac.in \\ ${ }^{3}$ S.O.S. in Electronics and Photonics, Pt. R. S. University, Raipur, India, Email: kavithakur67@ gmail.com \\ ${ }^{4}$ Department of Electrical Engineering, University of Texas at Arlington, Texas, Email: rao@uta.edu
}

\begin{abstract}
The quantization process is the most significant part of any image transform coder, which solely governs the loss characteristics during compression of the images. Hence, several quantization techniques have been developed to achieve better quantization performance for the image transform coders. The Optimum Non-negative Integer Bit Allocation (ONIBA) is one of the impotent techniques that provide optimal quantization bits based on the Variance characteristics of the transform coefficients for the best possible quantization in the image coders. However, the variance feature is not supposed to be the best indicator for all types of variations in the images. As a result, the performance of existing ONIBA techniques can be improved by the utilization of other feature that can accurately estimate the image activities. Therefore, this paper presents a new Gradient Feature-based ONIBA (GFONIBA) algorithm to achieve better quantization for the DCT based image transform coders as compared to the recent ONIBA algorithms. Extensive experiments are carried out to validate the quantization performance of the proposed GFONIBA algorithm. The results show that the proposed GFONIBA algorithm outperforms and provide a significant gain in the reconstructed image quality as compared to the recent ONIBA algorithms.
\end{abstract}

Key words: DCT based transform coding, Image compression, Optimum bit allocation, Quantization.

\section{INTRODUCTION}

Discrete Cosine Transform (DCT) based Transform Coding (TC) is the most important and popular technique for the signal coding and compression applications [1]-[3]. Usually, in the lossy version of DCT-TCs, the quantization process of the signal plays a crucial role and solely governs the reconstruction quality of the coder [4]. In past decade, several techniques have been developed to achieve better quantization of the DCT coefficients. Among the existing techniques, the Optimum Bit Allocation (OBA) is the most significant approach that provides the optimum bits for the quantization of DCT coefficients within the specified bit-budget [5].
Principally, the OBA technique tries to allocate the optimum number of bits for each of the quantizers to achieve maximum reduction in the Mean Square Error (MSE) between the original and reconstructed signal. Initially, the Lagrange multiplier method was used to obtain the required OBA solution for the TCs [5]. In Lagrange multiplier method, the optimal bit allocation is performed based on the Variance characteristics of the transform coefficients; in which the transform coefficient with higher variance will get higher bits as compared to the coefficients that have low variances. The final OBA solution obtained from the Lagrange multiplier method is real-valued optimum bits but for the practical cases, they must be non-negative integer values. Therefore, several techniques have been proposed in the literature to obtain the OBA solution in the form of Optimum Non-negative Integer Bits (ONIBs). The technique that provides the required ONIBs for the OBA problem is commonly known as Optimum Non-negative Integer Bit Allocation (ONIBA) Algorithm. The first ONIBA algorithm was proposed by Fox [6], which is also known as a greedy algorithm. Subsequently, Feber and Zeger [7] proposed a low complexity ONIBA algorithm for the TCs. Recently, in 2015, Hetam and Shirazi proposed a new version of the ONIBA algorithm, which not only offers the lowest computational complexity for the optimal bit generation but also addresses the zero variance conditions [8].

Moreover, similar to the Lagrange multiplier method, all the existing ONIBA algorithms strongly depend on the Variance characteristics of the transform coefficients for the bit allocation process. That is, from the very first Real-valued solution of the OBA problem to its current ONIBA solution, the Variance characteristics of the transform coefficients has been utilized as an image activity indicator. However, in 1999, Kim et al. [9], demonstrated that the variance feature provides a poor statistical measure of the image activity and hence it has a weak impact on the bit allocation process. Consequently, we can improve the quantization performance of the existing ONIBA algorithms by the proper selection of the image activity feature. Therefore, in this work, the authors present an improved ONIBA algorithm that utilizes image gradient as an input image activity feature in place of the 
conventional variance feature. The ultimate aim of the proposed Gradient Feature-based ONIBA (GFONIBA) algorithm is to achieve the better quantization performance for the DCT based image transform coders. The basic idea is to replace the variance feature based bit allocation process by the gradient feature based bit allocation. Hence, initially, the proposed gradient feature is applied for the Lagrange multiplier based optimal bit allocation and then subsequently applied to the recent ONIBA algorithm proposed by Hetam and Shirazi [8]. Several experiments are then performed to evaluate the quantization performance of the proposed GFONIBA algorithm against the recent ONIBA algorithm of [8] on the ground of two popular image quality indexes PSNR and MSSIM. The results obtained for the three standard test images show that the proposed GFONIBA algorithm provides much better quantization of the DCT coefficients as compared to the existing ONIBA techniques.

\section{PROPOSED GRADIENT FEATURE BASED ONIBA (GFONIBA) ALGORITHM FOR THE DCT BASED IMAGE TRANSFORM CODER}

This section first presents a detailed formulation of the OBA problem for the DCT based image transform coders. In the next step, we present the mathematical description of the Lagrange multiplier based optimum bit allocation technique which is the first OBA solution. In the last part of this section, we present the brief description of the recent ONIBA technique along with the development of the proposed GFONIBA algorithm.

Principally, in the DCT based image transform coders such as the popular JPEG coder [4], an input image is first divided into Non-Overlapping Blocks (NOBs) of size $8 \times 8$, and each NOB is then transformed by the $8 \times 8$ DCT transform. Finally, all the DCT transformed NOBs are quantized and entropy coded for the transmission or storage purpose. The block diagram representation of the baseline JPEG codec for the compression of grayscale images is shown in Figure 1.

Further, since the quantization process is the most critical part of the DCT based image transform coder which is mainly responsible for the loss of information during lossy compression. Thus, first, it is important to properly model the input and output relationship of the DCT quantizers. In case of the DCT based coders, the NOBs of size $8 \times 8$ are treated as an independent source for the quantization process. Hence, the total inputs available for the quantization are limited to $8 \times 8=64$, which requires $M=64$ scalar quantizers to accomplish the quantization of a DCT transformed NOB. Let us define the inputs available for the quantization as [5],

$$
F=\left[f_{1}, f_{2} \ldots \ldots . f_{M}\right]
$$

Where, $f_{1}, f_{2} \ldots \ldots f_{M}$, are the DCT coefficients to be quantize that are taken in raster scan order form a DCT NOB. Next, to quantize the input source given in (1) we need $k=1,2, \ldots, M$ uniform scalar quantizers. Let us first determine an expression for the distortion, and then we can find the bit allocation that minimizes the distortion using the conventional Lagrange multiplier method [5]. Suppose, $R$ is the average number of bits per sample given to the encoder for the quantization of DCT coefficients and if the encoder used $R_{k}$ number of bits for the quantization of the $k^{\text {th }} \mathrm{DCT}$ coefficient, then

$$
R=\frac{1}{M} \sum_{k=1}^{M} R_{k}
$$

The reconstruction error variance for the $k^{\text {th }}$ quantizer $\sigma_{r k}^{2}$ is related to its input variance $\sigma_{\theta_{k}}^{2}$ by [5],

$$
\sigma_{r_{k}}^{2}=\alpha_{k} 2^{-2 R_{k}} \sigma_{\theta_{k}}^{2}
$$

Where, $\alpha_{k}$ is a factor that depends on the distribution of input and the characteristics of the quantizers. The total reconstruction error is then give by,

$$
\sigma_{r}^{2}=\sum_{k=1}^{M} \alpha_{k} 2^{-2 R_{k}} \sigma_{\theta_{k}}^{2}
$$

Equation (4) provides a clear relationship between the number of bits $R_{k}$ used for the quantization of $k^{\text {th }}$ DCT coefficient and the total distortion generated due to the quantization of total $M$ transform coefficients. With this definition of the total distortion, we can define the objective of the bit

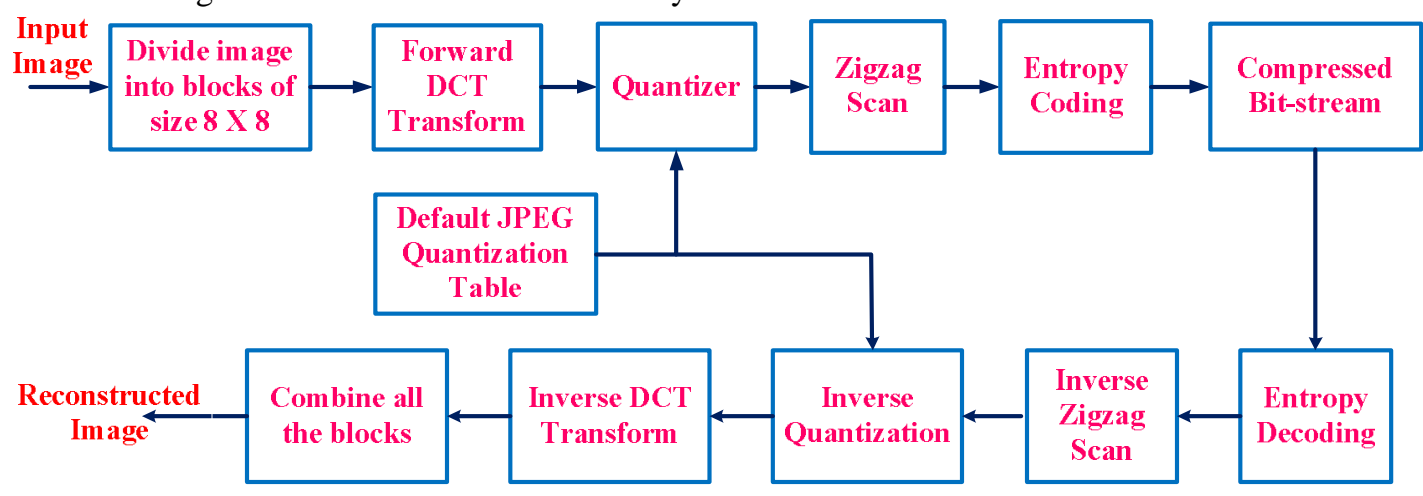

Figure 1: Block diagram of baseline JPEG codec [4]. 
allocation process as to determination the value of $R_{k}$ to minimize (4) with the constraint of (2). Suppose, $\alpha_{k}$ is a constant value $\alpha$ for all $k$, then we can frame the minimization problem given in (4), in terms of Lagrange multiplier as [5],

$$
\gamma=\alpha \sum_{k=1}^{M} \alpha_{k} 2^{-2 R_{k}} \sigma_{\theta_{k}}^{2}-\lambda\left(R-\frac{1}{n^{2}} \sum_{k=1}^{M} R_{k}\right)
$$

For the minimization of (5), let us take the derivative of $\gamma$ with respect to $R_{k}$ and set it equal to zero, it will provide an expression for $R_{k}$ as,

$$
R_{k}=\frac{1}{2} \log _{2}\left(2 \alpha \operatorname{In} 2 \sigma_{\theta_{k}}^{2}\right)-\frac{1}{2} \log _{2}\left(\frac{\lambda}{M}\right)
$$

Using (2) and (6), the final OBA solution obtained from the Lagrange multiplier method can be given by,

$$
R_{k}=R+\frac{1}{2} \log _{2}\left[\frac{\sigma_{\theta_{k}}^{2}}{\prod_{k=1}^{M}\left(\sigma_{\theta_{k}}^{2}\right)^{\frac{1}{M}}}\right]
$$

Equation (7) gives the individual bits $R_{k}$ for $k=1,2, \ldots, M$, quantizers that will minimize (5) within the constraint of (2). However, the values of $R_{k}$ obtained from (7) are not necessarily integers or even positive values. While for the practical quantization of DCT coefficients they must be positive integer values. Hence, to addresses this issue several Optimum Non-negative Integer Bit Allocation (ONIBA) algorithms have been proposed [6]-[8]. All the existing ONIBA algorithms are recursive in nature, and similar to the Lagrange multiplier method, they all strongly rely on the source variances for the final bit allocation. The general steps of the ONIBA algorithm are as follows,

Step 1: Determine the source variances $\sigma_{\theta_{k}}^{2}$ for each transform coefficient.

Step 2: Initially set the reconstruction error variances $\sigma_{r_{k}}^{2}=\sigma_{\theta_{k}}^{2}$, and set $R_{k}=0$ for all $k$, and also set the total bits available for encoding $B=M R$.

Step 3: Sort the variances $\sigma_{r_{k}}^{2}$ in ascending order and set the maximum variance as $\sigma_{m_{k}}^{2}$.

Step 4: Increment the corresponding $R_{k}$ for the $\sigma_{m_{k}}^{2}$ by one, then estimate the reduction factor in $\sigma_{m_{k}}^{2}$. Finally reduce the $\sigma_{m_{k}}^{2}$ by the obtained reduction factor.

Step 5: Decrement $B$ by one. If $B=0$ then stop; otherwise go to step 4.

The above steps present a general procedure of the existing ONIBA algorithms. However, most of these steps are depend on the nature of a particular ONIBA algorithm. The detailed description of the popular ONIBA algorithms can be seen in [6]-[8].

\section{PROPOSED GFONIBA ALGORITHM}

The bit allocation techniques described in the first part of this section clearly reveals that both the existing techniques viz. the Lagrange multiplier method and the ONIBA algorithms strongly depend on the source variances to obtain the desired OBA solutions. That is all the existing OBA solutions are obtained based on the prior assumption that the variance feature is a strong indicator for the image variations. However, in 1999, Kim et al. [9], verified that the variance feature provides a poor statistical measure of the image activity and hence it has a weak impact on the bit allocation process. Therefore, this paper proposes an improved ONIBA algorithm that utilizes gradient feature as an image activity measure in the place of conventional variance feature. The ultimate aim of the proposed Gradient Feature-based ONIBA (GFONIBA) algorithm is to achieve better quantization for the DCT based image transform coders as compared to the existing ONIBA algorithms.

The proposed Gradient Feature (GF) is actually the mean gradient of a two-dimensional DCT block of size $n \times n$, which is defined as,

$$
\begin{aligned}
\Psi=\frac{1}{n^{2}}\left\{\sum_{i=1}^{8} \sum_{j=1}^{8} \sqrt{|f(i, j)-f(i+1, j)|}\right. \\
\left.+\sum_{i=1}^{8} \sum_{j=1}^{8} \sqrt{|f(i, j)-f(i, j+1)|}\right\}
\end{aligned}
$$

Where, $\Psi$ is the proposed gradient feature, $f(i, j)$ is the DCT coefficient in the NOB at the spatial position $i, j$ and $n$ (usually for DCT based coder $n=8$ ) is the size of the block.

Further, to obtain the required OBA solution using the proposed gradient feature, we need a proper model which can relate reconstruction error gradient to the source gradients. In order to establish such relationship, let us consider the same environment as used for the Lagrange multiplier method. Then, the relationship between the reconstruction error gradient for the $k^{\text {th }}$ quantizer $\Psi_{r_{k}}$ to its input gradient $\Psi_{\theta_{k}}$ can be given by,

$$
\Psi_{r_{k}}=\alpha_{k} 2^{-2 R_{k}} \Psi_{\theta_{k}}
$$

Where, $\alpha_{k}$ is again a factor that depends on the distribution of the source and the nature of the quantizer. The total reconstruction error is then given by,

$$
\Psi_{r}=\sum_{k=1}^{M} \alpha_{k} 2^{-2 R_{k}} \Psi_{\theta_{k}}
$$

Next, by assuming $\alpha_{k}$ is a constant value $\alpha$ for all $k$, we can obtain the solution of the proposed gradient feature based OBA problem given in (10) under the constraint of (2) using the conventional Lagrange multipliers method as, 
Vikrant Singh Thakur et al., International Journal of Networks and Systems, 7(6), August - September 2018, 1 - 7

$$
R_{k}=R+\frac{1}{2} \log _{2}\left[\frac{\Psi_{\theta_{k}}}{\prod_{k=1}^{M}\left(\Psi_{\theta_{k}}\right)^{\frac{1}{M}}}\right]
$$

Equation (11) gives the individual bits $R_{k}$ for $k=1,2, \ldots, M$, quantizers to minimize (10) within the constraint of (2). However, similar to the OBA solution given in (7), the values of $R_{k}$ obtained from (11) are not necessarily integers or even positive values. But the formulation obtained in (11) ensures that the proposed gradient feature can be utilized for the optimal bit generation process. Hence, the next part of this section presents the development of the proposed Gradient Feature-based ONIBA (GFONIBA) algorithm.

Suppose the total number of bits assigned to the encoder is $B=M R$, then (11) can be modified as,

$$
R_{k}=\frac{B}{M}+\frac{1}{2} \log _{2}\left[\frac{\Psi_{\theta_{k}}}{\prod_{k=1}^{M}\left(\Psi_{\theta_{k}}\right)^{\frac{1}{M}}}\right]
$$

The solution $R_{k}$ obtained from (12), becomes an ONIBA solution if it belongs to a non-negative positive integer class $\mathrm{Z}^{+}$. In that case, we can refer this solution as $R_{k}^{*+}$, and the GFONIBA problem can be framed as: determine the values of $R_{k}^{*+}$, for $k=1,2, \ldots, M$ quantizers to minimize (12) with the constraint of (2).

In order to obtain the desired GFONIBA solution, it is impotent to properly handle the negative bit allocation problem of the conventional Lagrange method. One possible solution is the removal of the source corresponding to the negative bit allocation with the zero quantization bit. The following theorem provides a clear visualization of the source removal and the new bit allocation process for the remaining sources [8].

Theorem 1: Let $U_{0}=\{1,2 \ldots, k\}$ is a set of input sources in an ONIBA problem. If we remove the $j^{\text {th }}$ source from $U_{0}$, then for the new set of sources $U_{\text {new }}=U_{0} \backslash\{j\}$, (where $\backslash$ denotes the set minus operation), the optimal number of integer bits for source $k, R_{k}^{*}\left(U_{\text {new }}\right)$ can be obtained as,

$$
R_{k}^{*}\left(U_{\text {new }}\right)=R_{k}^{*}\left(U_{0}\right)+R_{j}^{*}\left(U_{0}\right) /(M-1)
$$

Proof: Let us first define $G\left(U_{0}\right)$ as the geometric mean of the gradients of DCT coefficients which is given as,

$$
G\left(U_{0}\right)=\prod_{k=1}^{M}\left(\Psi_{\theta_{k}}\right)^{\frac{1}{M}}
$$

Then (12) becomes,

$$
R_{k}^{*}\left(U_{0}\right)=\frac{B}{M}+\frac{1}{2} \log _{2}\left[\frac{\Psi_{\theta_{k}}}{G\left(U_{0}\right)}\right]
$$

Now, by the removal of $j^{\text {th }}$ source from $U_{0}$ the new geometric mean and new bit allocation are given by,

$$
\begin{aligned}
& G\left(U_{\text {new }}\right)=\left(G\left(U_{0}\right)^{M} / \Psi_{j}\right)^{1 / M-1} \\
& R_{k}^{*}\left(U_{\text {new }}\right)=\frac{B}{M-1}+\frac{1}{2} \log _{2}\left[\frac{\Psi_{\theta_{k}}}{G\left(U_{\text {new }}\right)}\right]
\end{aligned}
$$

Substituting (16) into (17), we get

$$
\begin{aligned}
R_{k}^{*}\left(U_{\text {new }}\right)= & \frac{B}{M-1}+\frac{1}{2} \log _{2}\left[\frac{\Psi_{\theta_{k}}}{\left(G\left(U_{0}\right)^{M} / \Psi_{j}\right)^{1 / M-1}}\right] \\
= & \frac{B}{M-1}+\frac{1}{2} \log _{2}\left[\frac{\Psi_{\theta_{k}} \Psi_{\theta_{k}}^{2 / M-1}}{G\left(U_{0}\right)^{1+1 / M-1}}\right] \\
R_{k}^{*}\left(U_{\text {new }}\right)= & \frac{B}{M}+\frac{B}{M(M-1)}+\frac{1}{2} \log _{2}\left[\frac{\Psi_{\theta_{k}}}{G\left(U_{0}\right)}\right] \\
& +\frac{1}{2(M-1)} \log _{2}\left[\frac{\Psi_{\theta_{j}}}{G\left(U_{0}\right)}\right]
\end{aligned}
$$

Finally form (15) and (17) we have,

$$
R_{k}^{*}\left(U_{\text {new }}\right)=R_{k}^{*}\left(U_{0}\right)+R_{j}^{*}\left(U_{0}\right) /(M-1)
$$

Next, let us define $U^{\prime}(m)=U_{0} \backslash\{1,2, \ldots, m\}=\{m+1, m+2, \ldots, M\}$, where $U^{\prime}(0) \square U_{0}=\{1,2, \ldots, M\}$, then for any $k>m$,

$$
R_{k}^{*}\left(U^{\prime}(m)\right)=R_{k}^{*}\left(U_{0}\right)+\sum_{j=1}^{m} R_{j}^{*}\left(U^{\prime}(j-I)\right) /(M-j)
$$

Equation (19) can be easily obtained by sequentially repeating (18) for $j=1$ to $m$. Now, if the sources are sorted with respect to their gradient values $\Psi_{\theta_{k}}$, such that for $m=1,2, \ldots . j$-1 we have $R_{m}^{*}\left(U^{\prime}(j-1)\right)<0$. Then

$$
R_{j}^{*+}= \begin{cases}R_{j}^{*}\left(U^{\prime}(j-I)\right), & \text { if } R_{j}^{*}\left(U^{\prime}(j-I)\right) \geq 0 \\ 0, & \text { otherwise }\end{cases}
$$

Where, $R_{k}^{*}$ is the integer-valued solution, and $R_{k}^{*+}$ is the desired non-negative integer-valued solution of the proposed gradient feature based OBA problem.

Now, let us define an intermediate term as,

$$
B_{0}(m)=\sum_{j=1}^{m} R_{j}^{*}\left(U^{\prime}(j-l)\right) /(M-j)
$$

Then according to (19) we have

$$
R_{k}^{*}\left(U^{\prime}(m)\right)=R_{k}^{*}\left(U_{0}\right)+B_{0}(m)
$$

Starting with the initial assumption $B_{0}(0) \square 0$, we can recursively find the value of intermediate term $B_{0}(m)$ as,

$$
B_{0}(m)=B_{0}(m-1)+R_{m}^{*}\left(U^{\prime}(m-1)\right) /(M-m)
$$

Using (22) and (23) along with the interpretation of Theorem 1 , we now present the final steps of the proposed GFONIBA algorithm as given in Algorithm 1. The proposed GFONIBA algorithm shown in Algorithm 1 provides the desired ONIBA solution based on the proposed gradient feature. 
Vikrant Singh Thakur et al., International Journal of Networks and Systems, 7(6), August - September 2018, 1 - 7

Algorithm 1. Proposed GFONIBA Algorithm for the DCT based image transform coders.

Step 1: Check $\Psi_{\theta_{1}}, \Psi_{\theta 2, \ldots} \ldots, \Psi_{\theta_{k}}$ are non-zero. If some of $\Psi_{\theta_{k}}$ s are zero, set $R_{k}^{*_{+}}=0$ for the corresponding sources and set $k^{\prime}=$ number of remaining $\Psi_{\theta_{j}} \mathrm{~s}$. Then rename the rest of $\Psi_{\theta_{j}} \mathrm{~s}$ as $\Psi_{\theta_{1}}, \Psi_{\theta 2, \ldots}, \Psi_{\theta_{k}}$.

Step 2: Sort the remaining input sources to the quantizers with respect to their gradients $\Psi_{\theta_{k}}{ }^{\prime} s$ in the ascending order.

Step 3: Set $m=0, B_{0}(m)=0$, and compute $R_{k}^{*}$.

Step 4: While $R_{m+1}^{*}\left(U^{\prime}(m)\right)<0$ set $m=m+1$ and find $B_{0}(m)$ and $R_{m+1}^{*}\left(U^{\prime}(m)\right)$ recursively using (22) and (23).

Step 5: Set $n=m, U^{(n)}=\{n+1, \ldots, M\}$ and in Ref. 7 (Th. III.3) set $\boldsymbol{R}^{*}=\boldsymbol{R}^{*}\left(U^{(n)}\right)$ to find $\boldsymbol{R}^{*}\left(U^{(n)}\right)$. Then set $\boldsymbol{R}^{*+}=\boldsymbol{R}^{*}\left(U^{(n)}\right)$.

\section{RESULTS AND DISCUSSIONS}

This section presents an extensive performance evaluation of the proposed GFONIBA algorithm against the recent ONIBA algorithm given by Hetam and Shirazi [8] on the ground of image quality indexes PSNR [10]-[11] and MSSIM [12]. In order to test the practical quantization performance of both the algorithms, they have been tested using the baseline JPEG codec shown in Fig. 1. Meanwhile, for the proper evaluation of the quantization performance and its effect on the image compression, both the algorithms have been tested for three different greyscale test images each of size $512 \times 512$ as shown in Figure 2.

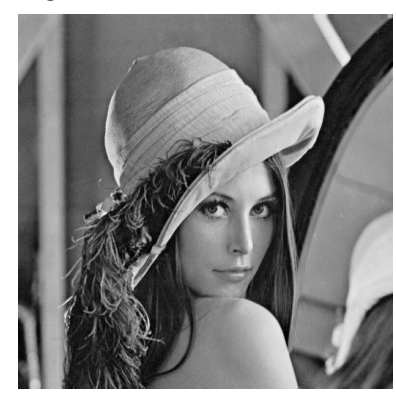

(a)

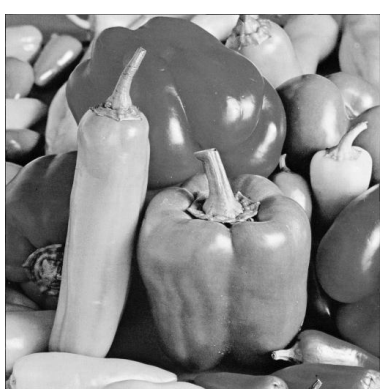

(b)

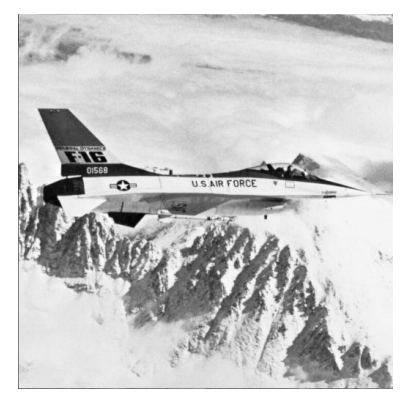

(c)

Figure 2: Standard grayscale test images, (a) Lena.png, (b) Peppers.png, and (c) F16_Jet.png.

Finally, after the compression and reconstruction of all the three test images on various compression rates (in bpp), the obtained values of PSNR and MSSIM parameters for the proposed GFONIBA algorithm and the ONIBA algorithm of Hetam and Shirazi [8], are tabulated in Table 1 to Table 6.

Table 1: PSNR comparison for test image Lena.

\begin{tabular}{|c|c|c|c|c|}
\hline \multirow[b]{2}{*}{ S. No. } & \multirow[b]{2}{*}{$\begin{array}{l}\text { Bitrate } \\
\text { in bpp }\end{array}$} & \multicolumn{2}{|c|}{ PSNR in $\mathrm{db}$} & \multirow[b]{2}{*}{$\begin{array}{c}\text { PSNR } \\
\text { Gain } \\
\text { in dB }\end{array}$} \\
\hline & & $\begin{array}{c}\text { ONIBA Algo- } \\
\text { rithm given by } \\
\text { Hetam and } \\
\text { Shirazi }[8] \\
\end{array}$ & $\begin{array}{c}\text { Proposed } \\
\text { GFONIBA } \\
\text { Algorithm }\end{array}$ & \\
\hline 1 & 0.2 & 28.204 & 29.107 & 0.903 \\
\hline 2 & 0.4 & 32.234 & 33.307 & 1.073 \\
\hline 3 & 0.6 & 34.535 & 35.640 & 1.105 \\
\hline 4 & 0.8 & 35.869 & 36.969 & 1.099 \\
\hline 5 & 1 & 37.045 & 38.168 & 1.123 \\
\hline 6 & 1.2 & 38.205 & 39.161 & 0.956 \\
\hline 7 & 1.4 & 38.885 & 39.909 & 1.024 \\
\hline 8 & 1.6 & 39.561 & 40.640 & 1.078 \\
\hline 9 & 1.8 & 40.471 & 41.394 & 0.923 \\
\hline 10 & 2 & 41.095 & 42.153 & 1.058 \\
\hline \multicolumn{4}{|c|}{ Average PSNR Gain in dB } & 1.034 \\
\hline
\end{tabular}

Table 2: PSNR comparison for test image Peppers.

\begin{tabular}{|c|c|c|c|c|}
\hline \multirow[b]{2}{*}{ S. No. } & \multirow[b]{2}{*}{$\begin{array}{l}\text { Bitrate } \\
\text { in bpp }\end{array}$} & \multicolumn{2}{|c|}{ PSNR in db } & \multirow[b]{2}{*}{$\begin{array}{l}\text { PSNR } \\
\text { Gain } \\
\text { in dB }\end{array}$} \\
\hline & & $\begin{array}{l}\text { ONIBA Algo- } \\
\text { rithm given by } \\
\text { Hetam and } \\
\text { Shirazi [8] } \\
\end{array}$ & $\begin{array}{c}\text { Proposed } \\
\text { GFONIBA } \\
\text { Algorithm }\end{array}$ & \\
\hline 1 & 0.2 & 27.271 & 28.765 & 1.494 \\
\hline 2 & 0.4 & 31.792 & 32.944 & 1.152 \\
\hline 3 & 0.6 & 33.661 & 34.695 & 1.034 \\
\hline 4 & 0.8 & 34.767 & 35.421 & 0.654 \\
\hline 5 & 1 & 35.577 & 36.301 & 0.725 \\
\hline 6 & 1.2 & 36.313 & 36.911 & 0.598 \\
\hline 7 & 1.4 & 36.799 & 37.434 & 0.635 \\
\hline 8 & 1.6 & 37.227 & 38.054 & 0.827 \\
\hline 9 & 1.8 & 37.655 & 38.766 & 1.111 \\
\hline 10 & 2 & 38.468 & 39.478 & 1.009 \\
\hline \multicolumn{4}{|c|}{ Averag } & 0.924 \\
\hline
\end{tabular}

Table 3: PSNR comparison for test image F16_Jet.

\begin{tabular}{|c|c|c|c|c|}
\hline \multirow[b]{2}{*}{ S. No. } & \multirow[b]{2}{*}{$\begin{array}{l}\text { Bitrate } \\
\text { in bpp }\end{array}$} & \multicolumn{2}{|c|}{ PSNR in db } & \multirow[b]{2}{*}{$\begin{array}{c}\text { PSNR } \\
\text { Gain } \\
\text { in dB }\end{array}$} \\
\hline & & $\begin{array}{c}\text { ONIBA Algo- } \\
\text { rithm given by } \\
\text { Hetam and } \\
\text { Shirazi }[8] \\
\end{array}$ & $\begin{array}{l}\text { Proposed } \\
\text { GFONIBA } \\
\text { Algorithm }\end{array}$ & \\
\hline 1 & 0.2 & 27.054 & 27.815 & 0.761 \\
\hline 2 & 0.4 & 31.288 & 32.608 & 1.320 \\
\hline 3 & 0.6 & 33.826 & 35.256 & 1.430 \\
\hline 4 & 0.8 & 35.609 & 37.110 & 1.501 \\
\hline 5 & 1 & 37.239 & 38.790 & 1.551 \\
\hline 6 & 1.2 & 38.429 & 39.996 & 1.567 \\
\hline 7 & 1.4 & 39.609 & 41.059 & 1.450 \\
\hline 8 & 1.6 & 40.425 & 42.011 & 1.586 \\
\hline 9 & 1.8 & 41.439 & 42.942 & 1.503 \\
\hline 10 & 2 & 42.382 & 43.873 & 1.491 \\
\hline \multicolumn{4}{|c|}{ Average PSNR Gain in dB } & 1.416 \\
\hline
\end{tabular}


Table 4: MSSIM index comparison for Lena image.

\begin{tabular}{|c|c|c|c|c|}
\hline \multirow[b]{2}{*}{ S. No. } & \multirow[b]{2}{*}{$\begin{array}{c}\text { Bitrate } \\
\text { in bpp }\end{array}$} & \multicolumn{2}{|c|}{ MSSIM index } & \multirow[b]{2}{*}{$\begin{array}{l}\text { Percentage } \\
\text { Gain in } \\
\text { MSSIM } \\
\text { index }\end{array}$} \\
\hline & & $\begin{array}{c}\text { ONIBA Algo- } \\
\text { rithm given by } \\
\text { Hetam and } \\
\text { Shirazi [8] }\end{array}$ & \begin{tabular}{|c|} 
Proposed \\
GFONIBA \\
Algorithm
\end{tabular} & \\
\hline 1 & 0.2 & 0.769 & 0.791 & 2.859 \\
\hline 2 & 0.4 & 0.868 & 0.879 & 1.290 \\
\hline 3 & 0.6 & 0.902 & 0.912 & 1.068 \\
\hline 4 & 0.8 & 0.920 & 0.928 & 0.797 \\
\hline 5 & 1 & 0.933 & 0.940 & 0.739 \\
\hline 6 & 1.2 & 0.944 & 0.948 & 0.470 \\
\hline 7 & 1.4 & 0.950 & 0.955 & 0.503 \\
\hline 8 & 1.6 & 0.956 & 0.961 & 0.593 \\
\hline 9 & 1.8 & 0.962 & 0.966 & 0.467 \\
\hline 10 & 2 & 0.966 & 0.971 & 0.496 \\
\hline \multicolumn{4}{|c|}{ Average percentage gain in the MSSIM } & 0.928 \\
\hline
\end{tabular}

Table 5: MSSIM index comparison for Peppers image.

\begin{tabular}{|c|c|c|c|c|}
\hline \multirow[b]{2}{*}{ S. No. } & \multirow[b]{2}{*}{$\begin{array}{c}\text { Bitrate } \\
\text { in bpp }\end{array}$} & \multicolumn{2}{|c|}{ MSSIM index } & \multirow[b]{2}{*}{$\begin{array}{l}\text { Percentage } \\
\text { Gain in } \\
\text { MSSIM } \\
\text { index }\end{array}$} \\
\hline & & $\begin{array}{c}\text { ONIBA Algo- } \\
\text { rithm given by } \\
\text { Hetam and } \\
\text { Shirazi [8] }\end{array}$ & $\begin{array}{l}\text { Proposed } \\
\text { GFONIBA } \\
\text { Algorithm }\end{array}$ & \\
\hline 1 & 0.2 & 0.734 & 0.760 & 3.544 \\
\hline 2 & 0.4 & 0.832 & 0.844 & 1.398 \\
\hline 3 & 0.6 & 0.863 & 0.873 & 1.188 \\
\hline 4 & 0.8 & 0.881 & 0.886 & 0.603 \\
\hline 5 & 1 & 0.895 & 0.902 & 0.817 \\
\hline 6 & 1.2 & 0.906 & 0.912 & 0.719 \\
\hline 7 & 1.4 & 0.914 & 0.920 & 0.722 \\
\hline 8 & 1.6 & 0.921 & 0.929 & 0.875 \\
\hline 9 & 1.8 & 0.929 & 0.939 & 1.111 \\
\hline 10 & 2 & \begin{tabular}{|c|}
0.938 \\
\end{tabular} & 0.949 & 1.141 \\
\hline \multicolumn{4}{|c|}{ Average percentage gain in the MSSIM } & 1.212 \\
\hline
\end{tabular}

Table 6: MSSIM index comparison for F16_Jet image.

\begin{tabular}{|c|c|c|c|c|}
\hline \multirow[b]{2}{*}{ S. No. } & \multirow[b]{2}{*}{$\begin{array}{l}\text { Bitrate } \\
\text { in bpp }\end{array}$} & \multicolumn{2}{|c|}{ MSSIM index } & \multirow[b]{2}{*}{$\begin{array}{l}\text { Percentage } \\
\text { Gain in } \\
\text { MSSIM } \\
\text { index }\end{array}$} \\
\hline & & $\begin{array}{l}\text { ONIBA Algo- } \\
\text { rithm given by } \\
\text { Hetam and } \\
\text { Shirazi [8] }\end{array}$ & \begin{tabular}{|l} 
Proposed \\
GFONIBA \\
Algorithm
\end{tabular} & \\
\hline 1 & 0.2 & 0.802 & 0.809 & 0.889 \\
\hline 2 & 0.4 & 0.887 & 0.896 & 1.103 \\
\hline 3 & 0.6 & 0.922 & 0.929 & 0.844 \\
\hline 4 & 0.8 & 0.940 & 0.946 & 0.668 \\
\hline 5 & 1 & 0.953 & 0.959 & 0.605 \\
\hline 6 & 1.2 & 0.962 & 0.965 & 0.386 \\
\hline 7 & 1.4 & 0.968 & 0.971 & 0.278 \\
\hline 8 & 1.6 & 0.972 & 0.976 & 0.372 \\
\hline 9 & 1.8 & 0.976 & 0.979 & 0.282 \\
\hline 10 & 2 & 0.980 & 0.982 & 0.261 \\
\hline \multicolumn{4}{|c|}{ Average percentage gain in the MSSIM } & 0.569 \\
\hline
\end{tabular}

From, the final PSNR comparisons shown in Table 1 to Table 3 , it is clearly evident that the proposed GFONIBA algorithm outperforms for all the three test images and provides average PSNR gains in the range of 0.9 to $1.5 \mathrm{~dB}$ as compared to the recent ONIBA algorithm developed by Hetam and Shirazi. Now, moving to the MSSIM index comparison shown from Table 4 to Table 6 , the proposed GFONIBA algorithm again provides better MSSIM values as compared to the recent ONIBA algorithm with the average percentage gain of about 0.5 to 1.2 percent.

The above comparative analysis clearly justifies that the proposed gradient feature based ONIBA algorithm can provide significantly better quantization of the DCT coefficient as compared to the existing ONIBA algorithms. Consequently, the proposed GFONIBA algorithm is a better alternative of the existing variance feature-based quantization techniques for the DCT based image transform coders.

\section{CONCLUSION}

In this paper, first, the OBA problem and its existing solutions are explored with the proper mathematical formulation. Thereafter, a new gradient feature based ONIBA algorithm has been developed to achieve best possible quantization for the DCT based image transform coders as compared to the existing variance feature based optimum bit allocation techniques. The results show that the proposed GFONIBA algorithm provides better quantization of the DCT coefficients, which results in higher PSNR and MSSIM values after reconstruction of the test images as compared to the recent ONIBA algorithms. Moreover, the proposed GFONIBA algorithm has explored the possibility that the quantization performance of transform coders can be greatly improved by the proper selection of image features that can accurately reflect the variation of the input image to be compressed.

\section{REFERENCES}

1. v. S. Thakur, S. Gupta, and K. Thakur. Hybrid WPT-BDCT transform for high-quality image compression, IET Image Processing, vol. 11, no. 10, pp. 899-909, 2017. https://doi.org/10.1049/iet-ipr.2016.0740

2. N. Ahmed, T. Natarajan, and K. R. Rao. Discrete cosine transform, IEEE Trans. Comm., vol. 23, no. 1, pp. 90-93, 1974.

3. K. R. Rao, and P. Yip. Discrete Cosine Transform: Algorithms, Advantages, Applications, 1st ed. Academic Press, Elsevier, 1990, ch.7, pp. 136-344. https://doi.org/10.1016/B978-0-08-092534-9.50013-8

4. G. K. Wallace. The JPEG still picture compression standard, Communication. ACM, vol. 34 , no. 4 , pp. 30-44, 1991.

https://doi.org/10.1145/103085.103089 
Vikrant Singh Thakur et al., International Journal of Networks and Systems, 7(6), August - September 2018, 1 - 7

5. K. Sayood. Introduction to Data Compression, 4th ed., Morgan Kaufman, San Francisco, CA, 2012.

6. B. Fox. Discrete Optimization via marginal analysis, Manage. Sci., vol. 13, no. 3, pp. 210-216, 1966.

https://doi.org/10.1287/mnsc.13.3.210

7. B. Farber, and K. Zeger. Quantization of multiple sources using nonnegative integer bit allocation, IEEE Trans. Inform. Theory, vol. 52, no. 11, pp. 4945-4964, 2006.

https://doi.org/10.1109/TIT.2006.883630

8. M. Hatam, and M. A. M. Shirazi. Optimum nonnegative integer bit allocation for wavelet-based signal compression and coding, Information Sciences, vol. 297, pp. 332-34, 2015.

https://doi.org/10.1016/j.ins.2014.11.019

9. Wook Joong Kim, Jong Won Yi and Seong Dae Kim. A bit allocation method based on picture activity for still image coding, IEEE Transactions on Image Processing, vol. 8, no. 7, pp. 974-977, Jul 1999.

https://doi.org/10.1109/83.772244

10. R. C. Gonzalez, R. E. Woods, Digital image processing, 3rd ed. Prentice Hall, 2012, ch. 8, pp. 526-538.

11. S. Jayaraman, S. Esakkirajan, and T. Veerakumar, Digital Image Processing, 1st ed., Tata McGraw Hill Education, 2009, ch. 9, pp. 444-541.

12. Z. Wang, et al. Image quality assessment: From error visibility to structural similarity," IEEE Transactions on Image Processing, vol. 13, no. 4, pp. 600-612, Apr. 2004.

https://doi.org/10.1109/TIP.2003.819861 